\title{
Plasmid copy number noise in monoclonal populations of bacteria
}

\author{
Jérôme Wong Ng, * Didier Chatenay, and Jérôme Robert ${ }^{\dagger}$ \\ Laboratoire Jean Perrin, FRE 3231 CNRS-UPMC, 24 rue Lhomond, 75005 Paris, France
}

\author{
Michael Guy Poirier \\ Department of Physics, Ohio State University, 191 W Woodruff Avenue, Columbus, Ohio 43210, USA
}

(Received 29 July 2009; revised manuscript received 27 October 2009; published 14 January 2010)

\begin{abstract}
Plasmids are extra chromosomal DNA that can confer to their hosts' supplementary characteristics such as antibiotic resistance. Plasmids code for their copy number through their own replication frequency. Even though the biochemical networks underlying the plasmid copy number (PCN) regulation processes have been studied and modeled, no measurement of the heterogeneity in PCN within a whole population has been done. We have developed a fluorescent-based measurement system, which enables determination of the mean and noise in PCN within a monoclonal population of bacteria. Two different fluorescent protein reporters were inserted: one on the chromosome and the other on the plasmid. The fluorescence of these bacteria was measured with a microfluidic flow cytometry device. We show that our measurements are consistent with known plasmid characteristics. We find that the partitioning system lowers the PCN mean and standard deviation. Finally, bacterial populations were allowed to grow without selective pressure. In this case, we were able to determine the plasmid loss rate and growth inhibition effect.
\end{abstract}

DOI: 10.1103/PhysRevE.81.011909

PACS number(s): 87.16.Yc

\section{INTRODUCTION}

Plasmids are extra chromosomal DNA fragments that constitute as much as $25 \%$ of some organisms' genetic material [1]. In bacteria, the genes coded on plasmids often transfer between different species, making them an interesting pool of shared genetic material [2]. In addition to genes expressed on the chromosome, plasmids provide many phenotypes to bacteria. Some of them are vital to the bacterium such as antibiotic resistance or toxin-antitoxin systems [3]. In order to replicate themselves, plasmids use the replication machinery of their hosts. These features allow the plasmids to exhibit interesting symbiotic behaviors with their host. Moreover, plasmids code for a biochemical network that regulates their replication frequency [4]. This regulation network, which varies from plasmid to plasmid, sets the plasmid copy number (PCN). Researches on plasmids have focused on unraveling and understanding the networks controlling the replication frequency [4]. For example, the use of noncoding RNA in regulation processes was first discovered in plasmids [5].

Fluctuations in plasmid replication frequency and timing or allocation of different copy numbers at cell division can cause the PCN to vary from bacteria to bacteria within an isogenic population. Within this framework, some quantitative models of PCN regulation evaluate the PCN distribution [6-8].

In order to understand these differences in PCN, it is important to determine both the mean and standard deviations of the PCN. On the experimental side, DNA titration is not a very precise measurement of the mean PCN. Quantitative

\footnotetext{
*Present address: Unité Génétique In Silico, Institut Pasteur 2528, rue du Dr Roux, 75015 Paris, France.

jerome.robert@lps.ens.fr
}

polymerase chain reaction (qPCR) may be more accurate [9] but does not give access to the standard deviation of the PCN when performed on a population. To our knowledge, no measurement of the PCN distribution is available in the literature.

In order to measure the PCN distribution in a population, many individual cell measurements of the PCN within a large bacterial population are required. One solution is in vivo labeling techniques. For example, insertion of a lac array into the plasmid and GFP-LacI fusion expression leads to fluorescent focal points [10] that can be counted. While these techniques proved suitable for extracting localization dynamics of chromosomes, they are problematic in cases involving higher numbers of objects for two reasons. First, the optical diffraction limits the number of distinguishable objects within a confined volume such as the bacterium cytoplasm. Second and more importantly, in cases of plasmids with low copy numbers between $5-10$, only $2-6$ focal points could be observed [11], which indicates that either plasmids spontaneously form clusters or fluorescent probes aggregate. In both cases, quantification of the PCN by focal point measurements will be erroneous.

Another approach is to measure the expression of a gene inserted in the plasmid to extract the PCN. However, both the PCN distribution and the noise in gene expression contribute to the expression distribution [12]. In order to diminish the noise sources, our strategy is to insert a second gene in the bacterial chromosome. With the exceptions of the noise in PCN and chromosomal copy number, the expression from this chromosomal gene is submitted to the same sources of noise as the plasmid gene. Hence, the expression distribution from a plasmid can be corrected by the expression noise from the chromosome, which allows us to determine information about the PCN distribution. More precisely, we constructed an E. Coli strain, in which the egfp [13] gene was inserted into the chromosome opposite to the chromosome ori to minimize copy number fluctuations dur- 
ing the cell cycle. The mOrange [14] gene was inserted in each of the studied plasmids. The egfp bearing strain was then transformed with each plasmid separately. We chose the mOrange protein because it is the brightest fluorescent protein within the orange-red spectrum. Moreover, it could be reasonably excited with the same wavelength as the enchanced green fluorescent protein (EGFP) . Both genes were under control of the same isopropyl-beta-thio-galactoside (IPTG) inducible PtacI promoter [15] and terminated by the same TR2 termination [16]. In order to perform measurements on individual cells at low fluorescence levels, we developed a microfluidic flow cytometer ( $\mu$ FACS) based on the work of Fu et al. [17]. Using the hypothesis described in Sec. III C, the analysis of the first and second moments of the expression of both genes within the whole population allows us to estimate the PCN mean and variance.

\section{MATERIALS AND METHODS}

\section{A. Strains}

We modified the Top10 (Clontech) strain, which is a derivative of Escherichia coli $K-12$ as follows: chromosomal copy of lacI was replaced by lacIq1 allele [18] by homologous recombination using the counterselection bacterial artificial chromosome (BAC) modification kit from Genebridges [19] (Genebridges GmbH, Dresden, Germany). The lacIq1 allele represses the PtacI promoter a hundred times more efficiently than the wild-type lacI. Using the same homologous recombination technique, EGFP expression construct was cloned at the opposite of the origin of replication, next to the pyrF gene (1 $339945-1340682$ bp).

\section{B. Expression system}

The EGFP expression system was constructed with the egfp gene, the PtacI promoter [15], and the TR2 termination sequence [16]. The egfp sequence was amplified from the plasmid pEGFP (Clontech). The promoter and the termination were added through two consecutive PCRs using overhanging oligonucleotides. The mOrange [14] expression system was constructed identically. mOrange gene was amplified from pRSET-BmOrange [14].

\section{Plasmids}

Four plasmids were studied: pZC320 [20] is a mini-F containing a sopABC partitioning system, pOU82 is a mini-R1-par ${ }^{-}$[21] without a partitioning system, pOAR64 is a mini-R1-par ${ }^{+}[22]$ with a parABS partitioning system, and pBR322 is a mini-ColE1. Both mini-R1 plasmids have the same temperature-sensitive origin of replication. In these plasmids, an mRNA coding for the replication initiator RepA can be expressed through a $\lambda$ promoter. This promoter is strongly inhibited by CI857, which is also constitutively expressed from the plasmid. At high temperature, CI857 is unstable, which results in high levels of RepA. The mOrange construct and the plasmids were assembled following standard restriction/ligation protocols. All plasmids were sequenced to verify proper insertion (Eurofins/MWG/Operon).
All enzymes used were from Fermentas and all purification kits were from Qiagen.

\section{Microfluidic device}

Molds containing a 2- $\mu \mathrm{m}$-height motif were made through usual soft lithography techniques with SU8-2002 photoresist (MicroChem) using chrome deposited masks (Dupont photomask). The motif, which was based on $\mathrm{Fu}$ et al. [17], consists of three channels joined in a Y-shape junction with branches $5 \mu \mathrm{m}$ in width. Elastomer (RTV615, GE Bayer) was poured into the mold and baked at $90{ }^{\circ} \mathrm{C}$ for $2 \mathrm{~h}$. The cross-linked elastomer was pealed and then drilled to make the reservoirs. The elastomer and the quartz cover slip were placed in a homemade plasma cleaner for about $5 \mathrm{~s}$. Then, the molded elastomer was sealed to the quartz cover slip. The chip was immediately filled with phosphate buffer saline (PBS, $p \mathrm{H}=7.4$ ). The bacterial culture of interest was placed in one of the reservoirs and allowed to flow under pressure within channels. Typical flow speed is 5 $\times 10^{-3} \mathrm{~ms}^{-1}$.

\section{E. Microscopy and data analysis}

The chip was placed on an inverted microscope (IX81, Olympus) and observations were performed using an oil immersion 63x Objective (PlanApo, Zeiss). Bacterial fluorescence was excited with a solid-state $488 \mathrm{~nm}$ laser (Sapphire 488 HP, Coherent). The laser light wave front was modified using a spatial light modulator (LCR-720, HOLOEYE Photonics $\mathrm{AG}$ ) to obtain a homogeneous restricted excitation area (Fig. 1). The flow channel was placed within this area. The light intensity was on the order of a few mW. Fluorescence was collected separately on green and orange channels. Light intensity was measured using avalanche photodiodes (SPCM-AQR-15, Perkin Elmer). TTL pulses from avalanche photodiodes were counted by an acquisition board (NI-PCI6602, National Instruments). The experiment was controlled using a custom-made LABVIEW interface (National Instruments). Considering the bacterial flow rate, fluorescent proteins are excited on a millisecond time scale. It is far too short to take into account any photobleaching effect. Data were analyzed using IGOR 5.1 and MATLAB 2006b programs.

\section{F. Quantitative PCR}

Bacteria were grown as described in the next section. The total DNA was extracted from a $1 \mathrm{ml}$ sample of bacteria bearing the various plasmids (Qiagen Qiamp). qPCR was performed on a light cycler (Roche Diagnosis) using SYBRgreen as a fluorescent intercalant agent (Qiagen). Oligonucleotides were designed to target two genes: bla and pyrF. All our plasmids contained the bla sequence, which codes for the ampicillin resistance. The pyrF sequence is located at the termini of the chromosome, next to the cloned egfp sequence. Five dilutions of each extract were amplified with the two sets of oligonucleotides. Data were analyzed with LIGHTCYCLER3 data analysis software.

\section{G. Bacterial culture and fluorescent protein expression}

Fresh overnight cultures from an isolated colony were diluted 500 times in Luria-Bertani (LB) medium supplemented 


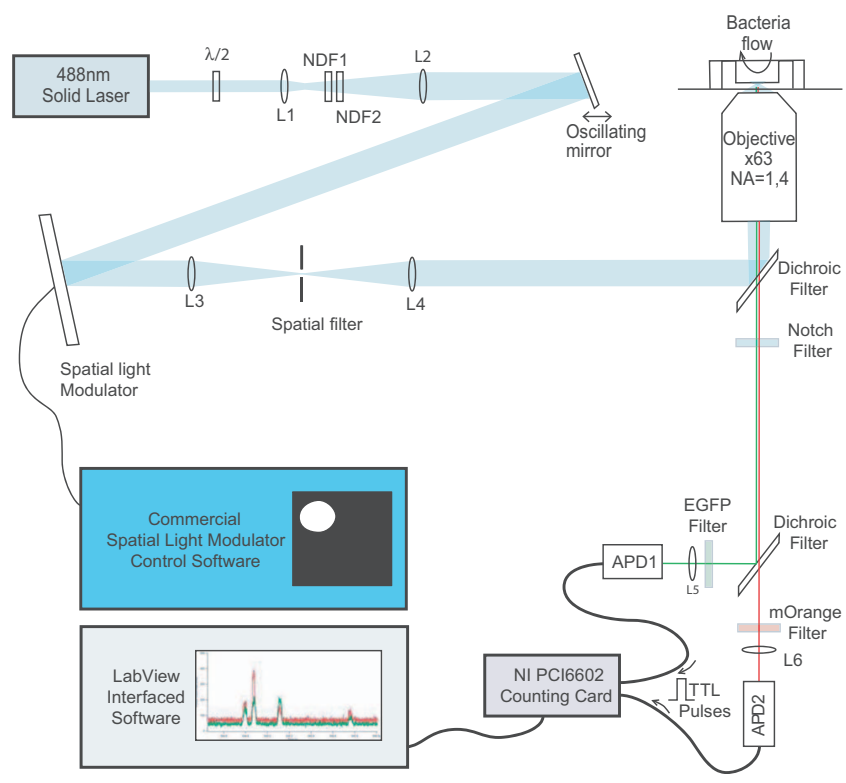

FIG. 1. (Color) The experimental setup is built on an inverted microscope. Laser light is shaped to a constant intensity disk of $\sim 20 \mu \mathrm{m}$ diameter in the field of view by a spatial light modulator (SLM). Fluorescent light is collected by the same objective and separated on green and orange channels through a combination of dichroic and emission filters. A subregion of the field of view (a $\sim 10 \mu \mathrm{m}$ disk) containing the channel through which the bacteria pass is focused on the avalanche photodiodes. TTL pulses from the avalanche photodiodes are fed to a counting acquisition board in a computer. A bacterium passing through the field of view causes an increase in the measured fluorescence intensity.

with the necessary antibiotics and grown at the chosen temperature with vigorous shaking $(200 \mathrm{rpm})$ up to an optical density at $600 \mathrm{~nm}\left(O D_{600}\right)$ between 0.20 and 0.25 . At this stage, the culture was diluted again by 100 times with fresh medium and grown up to an $O D_{600}=0.20-0.25$. IPTG was added to a final concentration of $1 \mathrm{mM}$ and the culture was incubated for another hour. At this point, chloramphenicol was added to a final concentration of $170 \mu \mathrm{g} / \mathrm{ml}$ to stop any additional translation of fluorescent protein. The cells were then washed into PBS with $170 \mu \mathrm{g} / \mathrm{ml}$ of chloramphenicol. Bacteria were placed back into the incubator at $37^{\circ} \mathrm{C}$ for further maturation of the fluorescent proteins overnight. The $p \mathrm{H}$ of the maturation medium is set to 7.4 by PBS. This value is suitable for both mOrange and EGFP stability. From this step on, care was taken to avoid unnecessary exposure to light in order to prevent photobleaching of both fluorescent proteins. The cells were washed again in PBS, filtered through a millipore syringe filter with a 5 - $\mu \mathrm{m}$-pore size to remove cell aggregates, and finally pipetted in the microfluidic chip. Typical fluorescence measurements required an hour long flow of bacteria for about $10^{4}$ detected events.

\section{CALIBRATION AND DATA ANALYSIS}

\section{A. Equal expression of fluorescent reporters}

Our data analysis relies on the fact that both EGFP and mOrange reporters are expressed identically from transcrip-
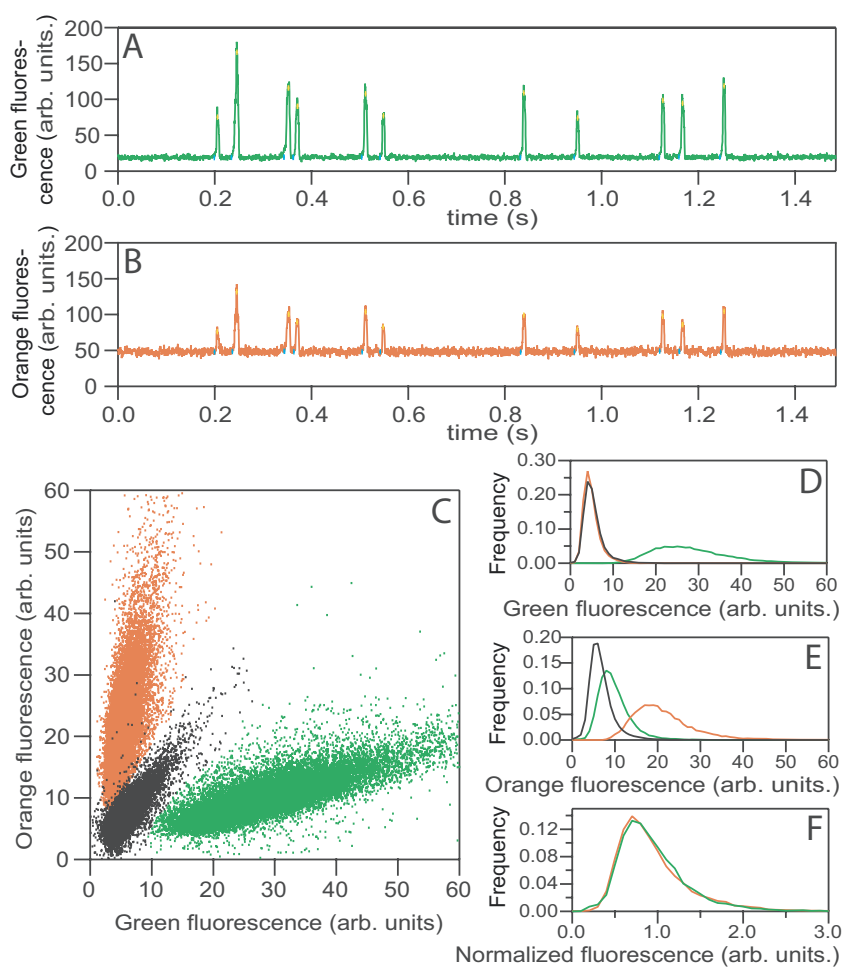

FIG. 2. (Color) [(a) and (b)] Temporal signal from the counting module, respectively, for the green and for the orange fluorescences. An abrupt increase is perceived when a bacterium passes through the detection field of the photon counting module. The peak and baseline of each event are spotted in blue and black, respectively. (c) Fluorescence scatter plot of three different populations. Bacteria bearing the green expression system on the chromosome without any plasmid is in green. Bacteria with the orange expression system on the same location as the previous strain also without a plasmid is in orange. The original strain bearing no fluorescence is in black. The leakage coefficients $\alpha$ and $\beta$ and the normalization factor $a$ (see Sec. III C) are deduced from the difference in mean fluorescence of those three populations. $\alpha$ could not be measured as it was too small $(<1 \%)$ and $\beta$ was estimated to $0.1674 \pm 0.0005$. $a$ was calculated to be $0.58 \pm 0.04$, which corrects for differences in optical pathways, fluorescent excitation, emission efficiency, and differences in expression. [(d) and (e)] Population histograms of green and orange fluorescence shown in (c). (f) Normalized histograms of EGFP and mOrange expressed from a mini-colE1 plasmid.

tion to protein folding. We tested this assumption by comparing EGFP expression to mOrange expression in the case of a mini-ColE1 plasmid. One mini-ColE1 plasmid was constructed with an EGFP expression system and another miniColE1 plasmid was constructed with an mOrange expression system. Chromosomal lacIql strains were transformed with these plasmids. Upon normalization of the mean, measured orange and green distributions superimposed (Fig. 2). We concluded that both fluorescent protein expressions have the same statistical properties.

\section{B. Autofluorescence and fluorescence leakage corrections}

In each optical channel, the measured fluorescence can be written as the sum of the fluorescence from the reporter of 
interest, the autofluorescence of the bacteria and the leakage from the other fluorescent reporter protein. We therefore write

$$
\begin{gathered}
F_{G}=P_{G}+A_{G}+\alpha P_{O}, \\
F_{O}=a P_{O}+A_{O}+\beta P_{G},
\end{gathered}
$$

where $F_{G}$ and $F_{O}$ are the measured fluorescence in green and orange, respectively, $P_{G}$ and $P_{O}$ are the fluorescence issued, respectively, from the EGFP and mOrange proteins. $\alpha$ and $\beta$ are the leak coefficients. $a$ is a constant coefficient, which normalizes orange and green excitation and detection efficiencies. It allows us to directly compare $P_{G}$ and $P_{O}$.

The parameters $\alpha, \beta$, and $a$ were estimated using bacteria exhibiting only one type of fluorescence (Fig. 2).

From Eqs. (1) and (2), moments of $P_{G}$ and $P_{O}$ can be written as functions of the moments of $F_{G}, F_{O}, A_{G}$, and $A_{O}$, assuming that the autofluorescence is uncorrelated with the expression of the fluorescent protein. For example, mean values $\left\langle P_{G}\right\rangle$ and $\left\langle P_{O}\right\rangle$ are written as

$$
\begin{aligned}
& \left\langle P_{G}\right\rangle=\frac{a}{a-\alpha \beta}\left\{\left(\left\langle F_{G}\right\rangle-\left\langle A_{G}\right\rangle\right)-\frac{\alpha}{a}\left(\left\langle F_{O}\right\rangle-\left\langle A_{O}\right\rangle\right)\right\}, \\
& \left\langle P_{O}\right\rangle=\frac{1}{a-\alpha \beta}\left\{\left(\left\langle F_{O}\right\rangle-\left\langle A_{O}\right\rangle\right)-\beta\left(\left\langle F_{G}\right\rangle-\left\langle A_{G}\right\rangle\right)\right\} .
\end{aligned}
$$

Moments of $A_{G}$ and $A_{O}$ were determined separately by measurements on nonfluorescent bacterial populations (black population in Fig. 2).

\section{Calculation of the mean and standard deviation of the plasmid copy number}

The mean and standard deviations of the PCN can be calculated rigorously from the measured expression of egfp and mOrange [23]. For simplicity, we present an approach based on a few basic assumptions. In particular, we suppose that the number of chromosomes and plasmids are kept constant during the induction time and that no cell division occurs. Moreover, we assume that all bacteria have the same cell cycle duration and that ages are uniformly distributed. Again, although these hypotheses are not valid in our experimental setup, the rigorous calculus leads to similar values. Global events, such as division or cell age, act on both expressions from the chromosome and from the plasmid. As we divide mean orange expression by mean green expression (see below), we get rid of most of these global fluctuations. We believe this is the underlying reason why the reductive case leads to values similar to the rigorous calculus.

Inside a bacterium, the green fluorescence intensity $P_{G}$ is the sum of the fluorescence of the EGFP expressed from each copy of the chromosome, that is,

$$
P_{G}=\sum_{i=1}^{n_{C}} P_{G}^{i}
$$

where $n_{C}$ is the number of copies of the chromosome and $P_{G}^{i}$ is the fluorescence of the EGFP proteins expressed from the $i$ th chromosome copy.
The same argument applies for the orange fluorescence $P_{O}$. It leads to

$$
P_{O}=\sum_{i=1}^{n_{P}} P_{O}^{i}
$$

where $n_{P}$ is the PCN and $P_{O}^{i}$ is the fluorescence of the mOrange proteins expressed from the $i$ th plasmid copy.

We assume that the expression from the chromosome has the same statistical properties as expression from the plasmid. For instance, the mean and standard deviations of the fluorescence of EGFP protein from one copy of the chromosome are equal to the mean and standard deviations of the fluorescence of mOrange protein from one copy of a plasmid. Then, taking the mean over the population, one gets

$$
n=\frac{\left\langle n_{P}\right\rangle}{\left\langle n_{C}\right\rangle}=\frac{\left\langle P_{O}\right\rangle}{\left\langle P_{G}\right\rangle},
$$

where $n$ is the mean PCN per chromosome, which only relies on our measurements and is independent of any other constant. It is important to note that we determine the average plasmid number per chromosome instead of the average plasmid number per cell. We are unable to directly deduce the number of plasmids and chromosomes from the measured green and red fluorescence intensities. This is because of the external noise in gene expression [12] and that the autofluorescence varies from cell to cell (Fig. 2).

We assume the replication of the plasmid to be independent of the replication of the chromosome and that $\left\langle n_{C}^{2}\right\rangle$ $=\left\langle n_{C}\right\rangle^{2}$. The solution for $\left\langle n_{P}^{2}\right\rangle$ then leads to the expression of the variance in $\mathrm{PCN}$,

$$
\sigma_{n_{P}}^{2}=\left\langle n_{P}\right\rangle\left\langle n_{C}\right\rangle\left\{1-\frac{\left\langle n_{P}\right\rangle}{\left\langle n_{C}\right\rangle}+\frac{1}{\left\langle P_{O} P_{G}\right\rangle}\left[\left\langle P_{O}^{2}\right\rangle-\frac{\left\langle n_{P}\right\rangle}{\left\langle n_{C}\right\rangle}\left\langle P_{G}^{2}\right\rangle\right]\right\} .
$$

One can then simply calculate the coefficient of variation or noise in the PCN by dividing the standard deviation by the mean

$$
\eta_{n_{P}}=\frac{\sigma_{n_{P}}}{\left\langle n_{P}\right\rangle} .
$$

Here, the noise only depends on the fluorescence measurement, whereas the calculation of the standard deviation needs the mean copy number of chromosome. Because this mean copy number varies a lot with the species and growth conditions [24], we will only discuss the noise in PCN $\eta_{n_{P}}$.

\section{RESULTS AND DISCUSSION}

\section{A. Expression is linear with gene copy number}

In order to verify the linearity of expression with gene copy number, two mOrange expression systems were cloned in the mini-F plasmid. Mean expression from the plasmid normalized to the expression from the chromosome scaled linearly to the number of mOrange constructs within the plasmid (Fig. 3). One might expect the expression from a single copy to lower with an increasing copy number due to 


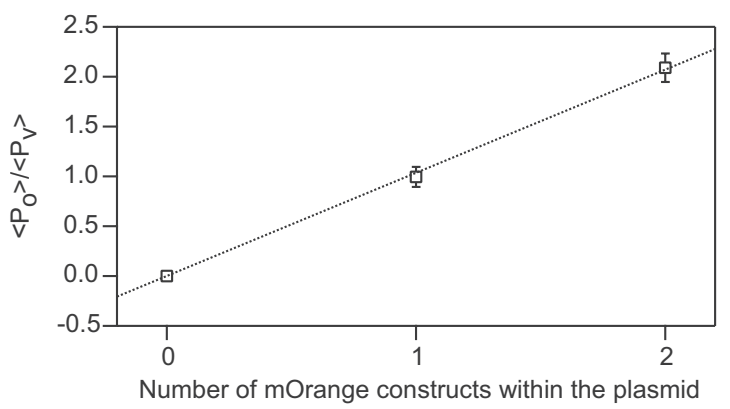

FIG. 3. Mean expression from the mini-F plasmid normalized to the expression from the chromosome as a function of the number of mOrange constructs in the mini-F plasmid. The line is a linear fit with a slope of $1.03 \pm 0.02$.

limited resources within the bacterium. However, this is not the case. The green fluorescence did not change significantly (Table I) whether the bacteria contained no plasmid or any plasmid of our series expressing mOrange. We therefore assume that the mean expression over a population is linear with respect to the gene copy number.

\section{B. Determination of the plasmid copy number mean and noise}

The mean PCN per chromosome for each plasmid was determined using formula (7). Results at $37{ }^{\circ} \mathrm{C}$ are summarized in Table I. Guyer et al. [25] measured a similar value for the mini-F. While no PCN measurement of our mini-R1 was reported, equivalent values were found for other derivatives of the R1 plasmid [26]. Finally, ColE1-based plasmids are generally accepted to have a PCN around 30 [27]. We measured a mean PCN three times higher for this plasmid. This discrepancy may result from measurement techniques as discussed below.

Expression from a plasmid seemed equivalent to the expression from the chromosome as $\left\langle P_{O}^{*}\right\rangle$ showed similar values to $n$. $n$ was chosen over $\left\langle P_{O}^{*}\right\rangle$ as a measurement of the PCN per chromosome because it integrates a reference within each experiment. PCNs estimated by qPCR are approximately a factor of 2 lower than values from fluorescence measurement for the mini-F and R1 plasmids, which increases to four times for the mini-colE1. Still, we are confident that our measurement is correct. The DNA extraction kit we used is specifically designed to extract chromosomal DNA. We tested that the extraction columns are less efficient for lighter DNA fragments while loaded with GeneRuler $1 \mathrm{~kb}$ DNA ladder (Fermentas). Therefore, we strongly suspect the DNA extraction process prior to the qPCR to favor high molecular mass. Finally, our bacterial preparation does not involve an extraction process or labeling efficiency. Since chromosome expression does not change for each plasmid, systematic overestimation of the mean PCN is unlikely. This makes our PCN measurements more reliable.

The major contribution of our work is the estimation of PCN noise. The mini-F showed a noise of about $50 \%$, which is consistent with having between 1 and 2 plasmid copies within each cell. Both mini-R1 had noise figures very close to simulations from Paulsson and Ehrenberg [8]. Concerning ColE1, Goss and Peccoud [28] evaluated by numerical simulations PCN noise of about $20 \%$, which fairly matches our $25 \pm 3 \%$.

\section{Par system reduces both mean and noise of plasmid copy number}

We observed a lower expression level from the mini-R1-par ${ }^{+}$than from the mini-R1-par ${ }^{-}$. One possible reason could be a gene silencing effect caused by the par system. Indeed, it is known that the par system induces silencing of the neighboring genes [29]. This phenomenon is thought to result from ParB proteins binding to the parS sequence and then spreading along the DNA. Our mini-R1-par ${ }^{+}$contains five iteron sequences, which are RepA protein binding sites. RepA binding prevents the polymerization of ParB on the DNA. It has been shown that genes separated from the parS by this same sequence display no silencing effect [29]. Therefore, we concluded that lower expression from the mini-R1-par ${ }^{+}$was due to a lower copy number.

The lowering of the plasmid copy number can occur for two nonexclusive reasons. The first one is not linked to the partitioning system but to the iteron sequence. The RepA protein needed to suppress silencing initiates replication of the mini-R1 plasmids. Hence, a sequestering of RepA by the iteron repeat sequence would lead to a lower replication frequency and would result in a reduced PCN. The second reason is directly caused by the par system. The partitioning

TABLE I. Expression and deduced PCN mean and noise values at $37{ }^{\circ} \mathrm{C}$. Each value is the mean of at least five experiments. The errors are the standard deviation between the different experiments. $P_{O}^{*}$ is the mOrange expression $\left\langle P_{O}\right\rangle$ divided by the mean mOrange expression from a strain containing the mOrange construct on the chromosome (Fig. 2). $n_{q P C R}$ is the measured mean PCN from the qPCR experiments.

\begin{tabular}{lcccc}
\hline \hline & mini-F & mini-R1-par & mini-R1-par & mini-ColE1 \\
\hline$\left\langle P_{G}\right\rangle$ & $27.1 \pm 0.8$ & $28.5 \pm 0.8$ & $26.5 \pm 0.4$ & $25.7 \pm 1.9$ \\
$\left\langle P_{O}\right\rangle$ & $27.0 \pm 0.9$ & $244 \pm 16$ & $173 \pm 8$ & $2167 \pm 89$ \\
$n=\left\langle P_{O}\right\rangle /\left\langle P_{G}\right\rangle$ & $1.0 \pm 0.04$ & $7.8 \pm 0.2$ & $6.5 \pm 0.3$ & $95 \pm 2$ \\
$\left\langle P_{O}^{*}\right\rangle$ & $1.1 \pm 0.2$ & $10 \pm 2$ & $7 \pm 1$ & $88 \pm 16$ \\
$n_{q P C R}$ & $0.5 \pm 0.3$ & $3 \pm 1$ & $4 \pm 1$ & $25 \pm 5$ \\
$10^{2} \eta_{n_{P}}$ & $46 \pm 2$ & $34 \pm 2$ & $29.2 \pm 0.6$ & $25 \pm 3$ \\
\hline \hline
\end{tabular}


system is known to reduce loss probability [30]. Typically, when the plasmid is lost, a bacterium containing a "high" copy number is born. If selection for the plasmid bearing bacteria occurs, the resulting population will have a greater mean PCN. Therefore, the lack of partitioning system increases the plasmid loss rate, resulting in an increase in mean PCN.

The mini-R1-par ${ }^{+}$also showed a reduction in PCN noise relative to the mini-R1-par ${ }^{-}$. The noise in PCN is mainly due to two phenomena. At the cell division, plasmids are not distributed equally between the daughter cells. This occurs either because plasmids are randomly distributed or simply because there is an uneven number of plasmids to distribute. Second, even in bacteria bearing the same number of plasmids at birth, the number of replication events and their timing can be different, which leads to a different PCN in the end. Both mini-R1 have the same replication system. As expected, we deduced it is the par system that reduces the noise in PCN.

\section{Temperature dependence of the plasmid copy number and its variability}

The mini-R1 PCN regulation networks in this study were designed to be temperature sensitive [26]. At temperatures below $37{ }^{\circ} \mathrm{C}$, these plasmids behave as regular low copy $\mathrm{R} 1$ plasmids, whereas at temperatures higher than $37^{\circ} \mathrm{C}, \operatorname{Rep} A$ expression is increased and results in increased or even uncontrolled plasmid replication [26]. This increase in mean PCN was estimated with single cell resistance to ampicillin [31].

We measured both mini-R1 PCN between $30{ }^{\circ} \mathrm{C}$ and $39{ }^{\circ} \mathrm{C}$ (Fig. 4). PCN slowly increased between $30{ }^{\circ} \mathrm{C}$ and $37{ }^{\circ} \mathrm{C}$ by $46 \%$ for the mini-R1-par ${ }^{+}$and by $16 \%$ for the mini-R1-par ${ }^{-}$. PCN increased abruptly at $39{ }^{\circ} \mathrm{C}$. This abrupt response was also reported by Uhlin and Nordström [31]. The par system lowered the PCN at all temperatures, although the difference is less significant at $39{ }^{\circ} \mathrm{C}$. The coefficient of variation or noise was nearly constant from 30 to $37{ }^{\circ} \mathrm{C}$, then had a twofold increase at $39{ }^{\circ} \mathrm{C}$ (Fig. 4). Furthermore, there was no visible effect of the par system at $39{ }^{\circ} \mathrm{C}$ on $\mathrm{PCN}$ noise. These results indicate that the par system is less effective as the PCN is increased.

All the plasmids had an increasing PCN with temperature increase (Fig. 4). This shows that temperature and duplication time are important parameters when considering the replication regulation process. It is even more important if the replication frequency is low: the mini-F PCN jumped from 0.5 to 2 and the mini-ColE1 PCN evolved from 76 to 100 . An interesting feature is that the "relaxed" plasmid ColE1 has the lowest noise in PCN (23-25\%), whereas the stringent mini-F plasmid has a relatively high noise in PCN $(37-55 \%$ ) (see Fig. 4). For the mini-F, the tendency is that an increase in copy number is associated with a diminution of noise. However, for the other plasmids, noise was nearly constant. This indicates that PCN noise is inherent to the regulation processes coded on each plasmid.

\section{E. Plasmid loss rate}

We also tested the stability of the studied plasmids on $\sim 100$ generations. Every plasmid that we used coded for the
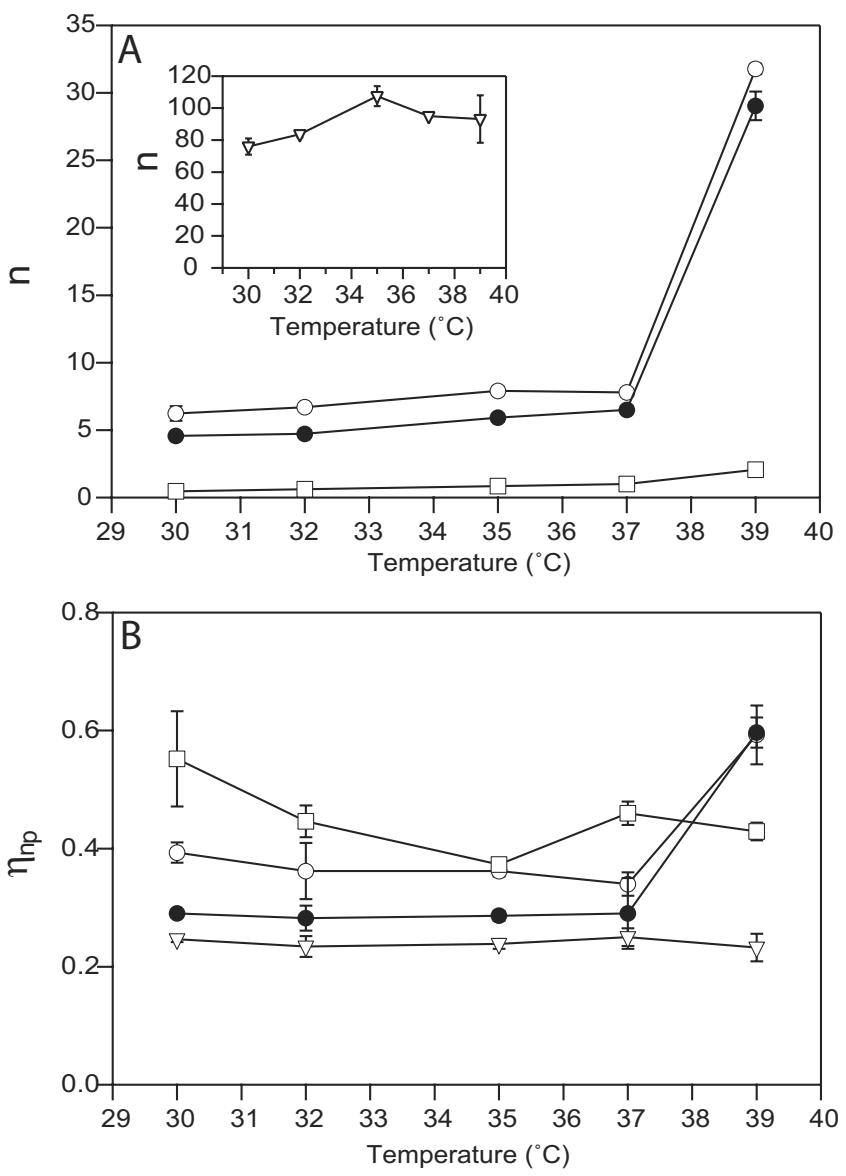

FIG. 4. Mean (a) and noise (b) in PCN for the mini-F (squares), the mini-ColE1 (triangle, inset), the mini-R1-par (open circle), and the mini-R1-par ${ }^{+}$(closed circle) as a function of temperature. The error bars are standard deviations obtained from multiple experiments. Error bars were not plotted whenever they were smaller than the data point size.

identical ampicillin resistance. Bacteria bearing plasmids were allowed to grow in a medium without ampicillin. A single colony was inoculated in LB medium and the culture was grown overnight at $37^{\circ} \mathrm{C}$. Each overnight culture was diluted 500 times. As the bacteria double their number at each generation, it takes $\log _{2}(500) \sim 9$ generations to overcome the dilution. Each strain was recultured every evening for ten days. We stored a $1 \mathrm{ml}$ sample of each overnight culture in glycerol at $-20{ }^{\circ} \mathrm{C}$. Prior to fluorescent measurements, the sample was thawed and cultured following the protocol described in Sec. II G. A bacterium exhibiting only EGFP fluorescence was considered cured from the plasmid. Experiments were performed on bacteria at generation 54 (five overnight cultures and induction process) and 99 (ten overnight and induction process).

The mini-F and the mini-R1-par ${ }^{+}$both contained a partitioning system and proved to be completely stable. The miniColE1 showed no plasmid loss on the time scale of our experiment. This is most likely a consequence of its high PCN. The only plasmid that proved unstable was the mini-R1-par ${ }^{-}$. We extracted the proportion $\Psi^{+}(g)$ at generation $g$ of bacteria harboring plasmids from our fluorescence measurements (see Fig. 5). As a control experiment, this pro- 

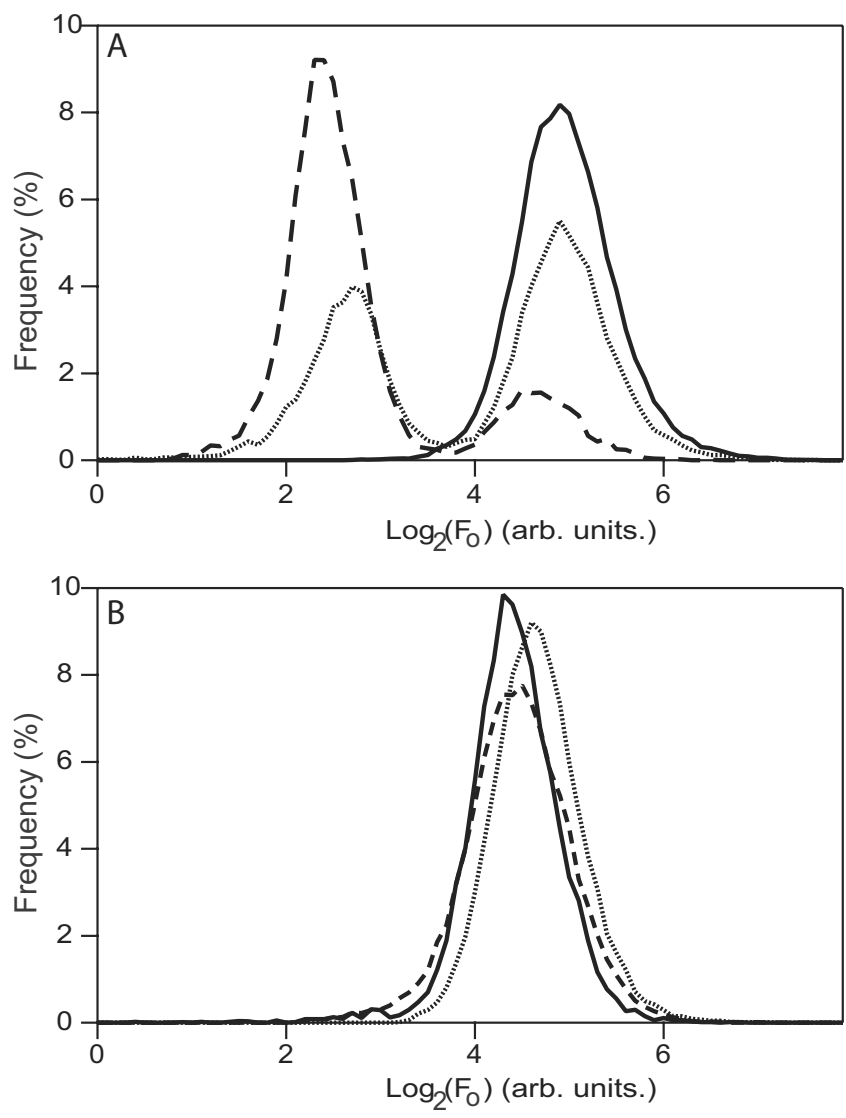

FIG. 5. Histograms of the orange fluorescence $\left(F_{O}\right)$ in logarithmic scale for the mini-R1-par- (a) and the mini-R1-par (b) at different generation times. In black (plain), the plasmids are in the presence of pressure selection. The red (dotted) and blue (dashed) histograms of the population are the fluorescence distributions after 54 and 99 generations in the medium without antibiotics, respectively.

portion was also evaluated by replica plating and proved to be consistent with fluorescent measurements.

Boe and Rasmussen [32,33] proposed two methods to calculate the plasmid loss rate. The first one assumes that the bacteria bearing plasmids and those without plasmids have the same division time. The proportion of bacteria cured from the plasmid $\Psi^{-}$increases through the generations solely because of the plasmid loss rate $\theta_{1}$, which should be the same at any generation. However, our estimations of $\theta_{1}$ from different generations gave different values, indicating that the second method should be used. The second method postulates a growth inhibition effect on plasmids bearing bacteria. This results in a different division time for bacteria bearing plasmids $\left(T^{+}\right)$and for cured bacteria $\left(T^{-}\right)$with $T^{+}>T^{-}$. Here, $\Psi^{-}$rises because of the plasmid loss rate $\theta_{2}$ but also because the cured bacteria grow faster. Our data analysis gave the same $\theta_{2}$ for the different generations $\left(\theta_{2}\right.$ $\simeq 0.53-0.57 \%$ at $37{ }^{\circ} \mathrm{C}$ ). Moreover, we were able to evaluate the growth inhibition effect as $T^{+} / T^{-} \simeq 1.066$, which corresponds to a difference in division time of about $2 \mathrm{~min}$.

\section{CONCLUSION}

We designed an experiment that allowed us to measure the mean and the standard deviations of PCN for a set of plasmids. The noise in PCN has been measured. We observed that the partitioning system decreased the PCN while also diminishing noise in PCN. We found that the mean PCN always varied with temperature regardless of the replication origin. Moreover, $\mathrm{PCN}$ noise showed either no or a relatively smooth change with temperature with the exception of the temperature-sensitive plasmids, for which noise increased abruptly at $39{ }^{\circ} \mathrm{C}$. Another feature is that random partitioning at high plasmid copy numbers induces less noise in PCN than low plasmid copy numbers with a partitioning system. Finally, we distinguished cured bacteria from plasmid bearing bacteria. This allowed us to measure the plasmid loss rate of the mini-R1-par- and the growth inhibition effect due to this plasmid.

Finally, it is known that the mean PCN of some plasmids can be changed by applying a different selection pressure such as antibiotic concentration [34]. Moreover, Spudich and Koshland suggested that variability in chemotactic response could be a source of adaptability on the population level [35]. With our experimental setup and analysis, we hope to address the question of whether the variability in PCN is a source of adaptability.

\section{ACKNOWLEDGMENTS}

J.W.N. acknowledges financial support from the Ministère de la Recherche and CNRS. This work was supported by the Grant No. 05-BLAN-0026-01 from the ANR.
[1] M. L. Holmes, F. Pfeifer, and M. L. Dyall-Smith, Gene 153, 117 (1995).

[2] C. Dahlberg, M. Bergstrom, M. Andreasen, B. Christensen, S. Molin, and M. Hermansson, Mol. Biol. Evol. 15, 385 (1998).

[3] K. Gerdes, L. K. Poulsen, T. Thisted, A. K. Nielsen, J. Martinussen, and P. H. Andreasen, New Biol. 2, 946 (1990).

[4] G. del Solar, R. Giraldo, M. J. Ruiz-Echevarría, M. Espinosa, and R. Díaz-Orejas, Microbiol. Mol. Biol. Rev. 62, 434 (1998).

[5] J. Tomizawa and T. Itoh, Proc. Natl. Acad. Sci. U.S.A. 78, 6096 (1981).
[6] K. Nordström and E. G. Wagner, Trends Biochem. Sci. 19, 294 (1994).

[7] P. J. Goss and J. Peccoud, Proc. Natl. Acad. Sci. U.S.A. 95, 6750 (1998).

[8] J. Paulsson and M. Ehrenberg, Q. Rev. Biophys. 34, 1 (2001).

[9] C. Lee, J. Kim, S. G. Shin, and S. Hwang, J. Biotechnol. 123, 273 (2006).

[10] A. S. Belmont, Trends Cell Biol. 11, 250 (2001).

[11] J. Pogliano, T. Q. Ho, Z. Zhong, and D. R. Helinski, Proc. Natl. Acad. Sci. U.S.A. 98, 4486 (2001).

[12] M. B. Elowitz, A. J. Levine, E. D. Siggia, and P. S. Swain, 
Science 297, 1183 (2002).

[13] R. Y. Tsien, Annu. Rev. Biochem. 67, 509 (1998).

[14] N. C. Shaner, R. E. Campbell, P. A. Steinbach, B. N. G. Giepmans, A. E. Palmer, and R. Y. Tsien, Nat. Biotechnol. 22, 1567 (2004).

[15] H. A. de Boer, L. J. Comstock, and M. Vasser, Proc. Natl. Acad. Sci. U.S.A. 80, 21 (1983).

[16] I. Gusarov and E. Nudler, Mol. Cell 3, 495 (1999).

[17] A. Y. Fu, C. Spence, A. Scherer, F. H. Arnold, and S. R. Quake, Nat. Biotechnol. 17, 1109 (1999).

[18] C. B. Glascock and M. J. Weickert, Gene 223, 221 (1998).

[19] Gene Bridges, Technical Protocol for Counter-Selection BAC Modification Kit (Advanced BAC Modification Kit), version 2.3 (2004).

[20] J. Shi and D. P. Biek, Gene 164, 55 (1995).

[21] K. Gerdes, J. E. Larsen, and S. Molin, J. Bacteriol. 161, 292 (1985).

[22] O. Rodionov and M. Yarmolinsky, Mol. Microbiol. 52, 1215 (2004).

[23] S. Ghozzi (unpublished).

[24] H. Bremer and P. P. Dennis, in Escherichia Coli and Salmo- nella, Cellular and Molecular Biology, edited by F. C. Neidhardt (ASM Press, Washington, DC, 1996), p. 1553.

[25] M. S. Guyer, R. R. Reed, J. A. Steitz, and K. B. Low, Cold Spring Harb Symp. Quant. Biol. 45, 135 (1981).

[26] J. E. Larsen, K. Gerdes, J. Light, and S. Molin, Gene 28, 45 (1984).

[27] J. Sambrook and D. W. Russel, Molecular Cloning: A Laboratory Manual, 3rd ed. (Cold Spring Harbor Laboratory Press, Cold Spring Harbor, NY, 2001).

[28] P. J. Goss and J. Peccoud, Pac. Symp. Biocomput 4, 65 (1999).

[29] O. Rodionov, M. Lobocka, and M. Yarmolinsky, Science 283, 546 (1999).

[30] S. Austin, S. Friedman, and D. Ludtke, J. Bacteriol. 168, 1010 (1986).

[31] B. E. Uhlin and K. Nordström, J. Bacteriol. 124, 641 (1975).

[32] L. Boe, Plasmid 36, 161 (1996).

[33] L. Boe and K. V. Rasmussen, Plasmid 36, 153 (1996).

[34] A. Løbner-Olesen, EMBO J. 18, 1712 (1999).

[35] J. L. Spudich and D. E. Koshland, Nature (London) 262, 467 (1976). 